\title{
PTSD and Suicidal Behaviors Amongst L'Aquila 2009 Earthquake Young Survivors
}

\author{
Claudia Carmassi ${ }^{1}$, Carlo Antonio Bertelloni ${ }^{1 *}$, Valerio Dell'Oste ${ }^{1}$, Chiara Luperini ${ }^{1,2}$, \\ Donatella Marazziti ${ }^{1}$, Rodolfo Rossi ${ }^{3}$ and Liliana Dell'Osso ${ }^{1}$ \\ ${ }^{1}$ Department of Clinical and Experimental Medicine, Section of Psychiatry, University of Pisa, Pisa, Italy, ${ }^{2}$ Department of \\ Experimental Medicine, University of L'Aquila, L'Aquila, Italy, ${ }^{3}$ Department of Systems Medicine, University of Rome Tor \\ Vergata, Rome, Italy
}

OPEN ACCESS

Edited by:

Umberto Volpe,

Marche Polytechnic University, Italy

Reviewed by:

Enrico Brosio,

Université de Caen

Normandie, France

Giuseppe Carrà,

University of Milano-Bicocca, Italy

${ }^{*}$ Correspondence:

Carlo Antonio Bertelloni

carlo.ab@hotmail.it

Specialty section

This article was submitted to

Public Mental Health,

a section of the journal

Frontiers in Public Health

Received: 02 August 2020

Accepted: 19 January 2021

Published: 10 February 2021

Citation:

Carmassi C, Bertelloni CA, Dell'Oste V, Luperini C, Marazziti D, Rossi R and Dell'Osso L (2021) PTSD and Suicidal Behaviors Amongst L'Aquila 2009

Earthquake Young Survivors.

Front. Public Health 9:590753.

doi: 10.3389/fpubh.2021.590753
Background: Post-traumatic stress disorder (PTSD) is one of the most frequent and severe psychiatric consequences of natural disasters, frequently associated with suicidality. The aim of this study was at examining the possible relationships between suicidal behaviors and full-blown or partial PTSD, in a sample of young earthquake survivors. The second aim was at investigating the specific role of PTSD symptoms on suicidality.

Methods: A total of 475 young adults who survived the L'Aquila 2009 earthquake, one of the most severe Italian disasters of the last decades, were recruited and assessed after 21 months from the catastrophe. Participants were evaluated by two questionnaires assessing subthreshold psychopathology, the Trauma and Loss Spectrum Self-Report (TALS-SR) to investigate both full and partial PTSD, and two specific Mood Spectrum Self-Report (MOODS-SR) sub-domains exploring suicidality, namely suicidal ideation and suicide attempts.

Results: The ensuing findings showed that suicidal ideation and suicide attempts were present, respectively, in 40 (8.4\%) and 11 (2.3\%) survivors. Rates of suicidal ideation were significantly more elevated in full-blown PTSD subjects (group 1), as compared with those suffering from partial (group 2) or no PTSD (group 3). Interestingly, group 2 subjects showed significantly more suicidal ideation than healthy individuals, and less than those of group 1, while the frequency of suicide attempts was similar across the three groups. Suicidal ideation was associated with higher scores in the following TALS-SR domains: grief-reactions, re-experiencing, avoidance and numbing, maladaptive coping, and personal characteristics/risk factor.

Conclusions: The results of the present study support and extend previous findings on the role of PTSD symptoms in suicidality after a severe earthquake. However, as compared with available literature, they also highlight the significant impact of sub-threshold PTSD manifestations in increasing the suicide risk in survivors of a mass disaster.

Keywords: natural disasters, earthquakes, post-traumatic stress disorder, suicidality, suicide attempts, suicidal ideation, trauma and loss spectrum-self report, mood spectrum-self-report 


\section{INTRODUCTION}

Earthquakes are one of the most frequent and disruptive natural disasters, as they expose involved population to not only injuries, devastation and death, but also severe psychological and psychiatric consequences. Indeed, during the past 40 years, a six-fold increase in the number of earthquakes worldwide has been reported, leading to an increased incidence and prevalence of different psychiatric disorders amongst survivors (1). Posttraumatic stress disorder (PTSD) is the most frequent psychiatric sequela following earthquakes, with prevalence rates ranging between 11.7 and $82.6 \%$ amongst survivors, depending on the characteristics of the population involved, the diagnostic criteria used to assess diagnosis and the traumatic event intensity (28). Suicidal behavior is not an uncommon consequence of earthquakes $(9,10)$, so that, not surprisingly, these natural disasters are widely investigating to assess their possible impact on this dramatic phenomenon (11). A great amount of data show increased rates of suicidal ideation and attempts, as well as of completed suicide, especially after highly destructive earthquakes (12-14). Again, PTSD seems to be one of the main factors linking a mass trauma to suicidality (15-19). A significant association between PTSD symptoms and suicide ideation emerged in about 2,300 child and adolescent survivors of the Wenchuan earthquake in China (17). Another study showed increased rates of PTSD and suicidal attempts, rather than depressive, or anxiety disorders, in a cohort of Swedish tsunami survivors, while suggesting that the effect of the trauma on suicidality was largely depending on stress-related disorders (18). The risk for suicidal behaviors, including suicidal ideations, plans, and attempts, in 1,369 adults exposed to an earthquake resulted to be about $11 \%$, with suicide attempts being related to the presence of PTSD (19).

L'Aquila 2009 earthquake, that occurred on April 6, 2009, is so called for its proximity to L'Aquila, the main town in the Abruzzi region of central Italy. The magnitude 6.3 tremor struck at 3:32 a.m. local time, damaging extensively the 13 th-century town, located only about 60 miles $(100 \mathrm{~km})$ northeast from Rome. As a whole, 309 people died, more than 1,600 were injured and approximately 66,000 were displaced to temporary settlements (9). Previous studies of our group on different samples of survivors from the L'Aquila 2009 earthquake, showed suicidality to be related to PTSD diagnosis, with rates of suicidal ideation and attempts ranging, respectively, between 7 and $12 \%$, and between 0.8 and $14 \%(4,20-22)$. Furthermore, some authors have criticized the current diagnostic criteria for PTSD, considering them too restrictive. The diagnostic and statistic manual for mental disorders (DSM-5) criteria for PTSD (23) diagnosis encompass the endorsement of four symptom clusters related to the traumatic event, particularly intrusion symptoms (criterion $\mathrm{B})$, avoidance (criterion C), negative alterations in cognitions and mood (criterion D), and alteration in arousal (criterion E). These diagnostic procedure could fail to include a large number of victims of traumatic events $(3,4,24,25)$. Indeed, despite only a few people exposed to a trauma suffer from a full-blow PTSD that can be easily recognized and treated, other individuals may develop PTSD symptoms classified as partial or subthreshold PTSD (26-28). This subthreshold psychopathology generally leads to a significant impairment, and it may require intervention and treatment (29-31). Various definition of partial PTSD have been reported in literature, so it could be difficult to compare findings from different studies. Accordingly, the prevalence of partial PTSD in earthquake survivors seems to range between 19 and $29 \%$ in the different studies $(3,4)$, possibly depending on the heterogeneous conceptualizations and diagnostic assessments. In the 2015, within the framework of the world health organization World Mental Health Surveys, some authors proposed a new definition for partial PTSD, according to the DSM-5 (23). McLaughlin et al. (27) proposed to define the presence of partial PTSD as the endorsement of two or three DSM- 5 criteria BE. Partial PTSD, similar to full-blown PTSD, has been related to increased suicidal behaviors, amongst earthquake survivors (3). Further, a comparable risk of suicide ideation in both full-blow and partial PTSD was also reported in a sample of 275 war veterans, while suggesting the importance to evaluate and to treat subthreshold trauma-related psychopathology (32). However, to the best of our knowledge, no studies utilized the new proposed criteria for partial PTSD in a sample of survivors from a natural disaster, such as an earthquake, or investigated their association with suicidality. Therefore, the present study was aimed at examining the possible relationships between suicide behaviors and either full-blow or partial PTSD, according to DSM-5 criteria, in a sample of L'Aquila 2009 earthquake survivors. The second aim was at investigating the specific role of PTSD symptoms on suicidality.

\section{METHODS}

\section{Study Participants}

The sample included 512 subjects recruited amongst highschool senior students who had experienced the L'Aquila 2009 earthquake, 21 months earlier. All subjects were directly exposed to the disaster and received assistance in emergency conditions. Full data were available for 475 out of the total of 512 subjects (94.2\% of the overall sample) (women: 203, 42.7\%; men: 272, $57.3 \%$; mean age $17.67 \pm 0.78$ years). Detailed characteristics of the study sample are reported elsewhere $(4,6)$.

The school council and the Ethics Committee of the University of L'Aquila approved all recruitment and assessment procedures. Eligible subjects or their legal representatives (for underage individuals) provided written informed consent to participate in the study that given was carried out in accordance with the declaration of Helsinki.

\section{Instruments and Assessments}

Participants were evaluated by two specific questionnaires, the so-called "Trauma and Loss Spectrum-Self Report (TALS-SR)" (33) to examine post-traumatic stress symptoms, and by a subscale of the "Mood Spectrum-Self Report (MOODS-SR)" (34) to investigate suicidal behaviors.

The TALS-SR encompasses 116 items, organized in nine domains, exploring the lifetime experience of a range of loss and/or traumatic events and lifetime symptoms, behaviors, and personal characteristics that could represent manifestations and/or risk factors for the development of a trauma or stressor 
related disorders. The nine domains explored by the TALS-SR are: loss events (I); grief reactions (II); potentially traumatic events (III); reactions to losses or upsetting events (IV); re-experiencing (V); avoidance and numbing (VI); maladaptive coping (VII); arousal (VIII); and personal characteristics/risk factors (IX). Items responses are coded in a dichotomous way (yes/no) and domain scores are obtained by counting the number of positive answers. To accomplish the aims of the present study, the instrument was adapted to examine symptoms related to L'Aquila 2009 earthquake. According to previous studies $(6,35)$, the presence of PTSD was assessed by means of TALS-SR items corresponding to DSM-5 criteria for PTSD diagnosis. Specifically, we utilized the following matching between symptom criteria and TALSSR items:

Criterion $\mathrm{B}(\mathrm{B} 1=80 ; \mathrm{B} 2=77 ; \mathrm{B} 3=79 ; \mathrm{B} 4=78 ; \mathrm{B} 5=81)$;

Criterion $\mathrm{C}(\mathrm{C} 1=86$; $\mathrm{C} 2=87$; and/or 88 and/or 89$)$;

Criterion D (D1 = 90; D2 = 95; D3 = 85; D4 = 96; D5 = 91; $\mathrm{D} 6=93$; $\mathrm{D} 7=92)$;

Criterion E (E1 = 108; E2 = 99 and/or 100 and/or 102 and/or

103 and/or 104; E3 = 106; E4 = 107; E5 = 105; E6 = 109).

To assess the presence of partial PTSD, we adopted the criteria proposed by previous studies corresponding to the endorsement of two or three symptom clusters $(27,36)$.

The MOODS-SR, a questionnaire exploring mood spectrum symptoms, includes 161 items coded as present/absent, for one or more periods of at least 3-5 days after the earthquake exposure. The items are organized into manic and depressive components as well as into a section that assesses disturbances in rhythmicity and vegetative functions, yielding a total of seven domains. In fact, both the manic and the depressive components are subtyped into three domains exploring mood, energy, and cognition symptoms, respectively. Suicidality was evaluated by means of six specific items of this questionnaire exploring both suicidal ideation and attempts after the earthquake exposure. The six MOODS-SR items combined to assess the suicidality are as it follows: "thought that life is not worth living" (item 102); "wished hel she would not wake up in the morning, or he/she would die in an accident or from something like a heart attack or a stroke" (item 103); "wanted to die or hurt him/herself" (item 104); "wanted to die and had a specific plan to hurt or kill him/herself" (item 105); "actually committed a suicide attempt" (item 106); and "committee a suicide attempt that required medical attention" (item 107). The first four items were combined in order to assess a new dichotomous variable named suicidal ideation that resulted to be endorsed when at least one of the items was endorsed. A second new dichotomous variable named suicidal attempts was derived from the endorsement of at least one out of the last two items (106 and/or 107).

\section{Statistical Analysis}

The Chi-square analysis was used to compare rates of suicidal ideation and suicidal attempts between subjects with PTSD, partial PTSD and without PTSD. Chi-square test was computed also to compare rates of suicidal ideation and suicidal attempts, between male survivors and female ones. In the subgroup of survivors with full or partial PTSD, the $t$-test was carried out to evaluate differences in TALS-SR domain scores between those with and without suicidal ideation. All statistical analyses were carried out using the Statistical Package for Social Science (SPSS Inc., Chicago 2006), version 22.0.

\section{RESULTS}

One hundred sixty-nine (35.6\%) and $202(42.5 \%)$ earthquake survivors were suffering from respectively, full and partial PTSD, according to DSM-5 criteria.

Rates of suicidal ideation and suicidal attempts in the total sample were, respectively, $8.4 \%(N=40)$ and $2.3 \%(N=11)$ (Figure 1).

Subjects with full-blown PTSD showed significantly higher suicidal ideation $(25,14.8 \%)$ than those with partial PTSD (14, $6.9 \% ; p=0.014)$ or those without PTSD $(1,1 \% ; p<0.001)$. Further, participants with partial PTSD showed significantly higher suicidal ideation than those without PTSD $(p=0.022)$. No statistically significant intergroup differences were found in suicidal attempts across the three groups. Rates of suicidal ideation were not statistically different between males and females [18 $(6.6 \%)$ vs. $22(10.8 \%), p=0.141]$ in the total sample. No gender differences emerged also for suicidal attempts [8 (2.9\%) vs. $3(1.5 \%), p=0.459]$.

In survivors with full or partial PTSD, the TALS-SR domains grief-reactions, re-experiencing, avoidance and numbing, maladaptive coping, and personal characteristics/risk factors scores were significantly more elevated in those subjects presenting suicidal ideation, as compared with those without it (Table 1).

\section{DISCUSSION}

To the best of our knowledge, this is the first study examining the relationship between DSM-5 full and partial PTSD and suicidal behavior in young survivors of the severe earthquake that occurred in 2009 in L'Aquila, the main town of a region from central Italy.

The ensuing findings revealed that the high-school senior students included in the present study showed suicidal ideation and suicidal attempt rates of, respectively, 8.4 and $2.3 \%$. Our results are in agreement with previous data on earthquake survivors reporting suicidal thoughts in around 5-19\% of subjects depending on the sampling selection and the severity of the catastrophe (37-41). Vehid et al. described $16.8 \%$ of suicidal ideations in 3,609 adolescents after 2 months from the Marmara earthquake. Another study detected a prevalence of suicidal ideation of $10.6 \%$ amongst 3,324 Chinese secondary school students exposed 1 month earlier to the Sichuan earthquake (38). On the contrary, other studies noted lower rates (0.4 and 3\%), although it should be underlined that suicidal behaviors were rarely assessed amongst adolescents and young adults exposed to natural disasters (42-44).

As expected, the analysis of the intergroup differences showed that survivors with DSM-5 partial PTSD had intermediate levels of suicidal ideation, that were significantly different in comparison with both those with or without PTSD. Our results 


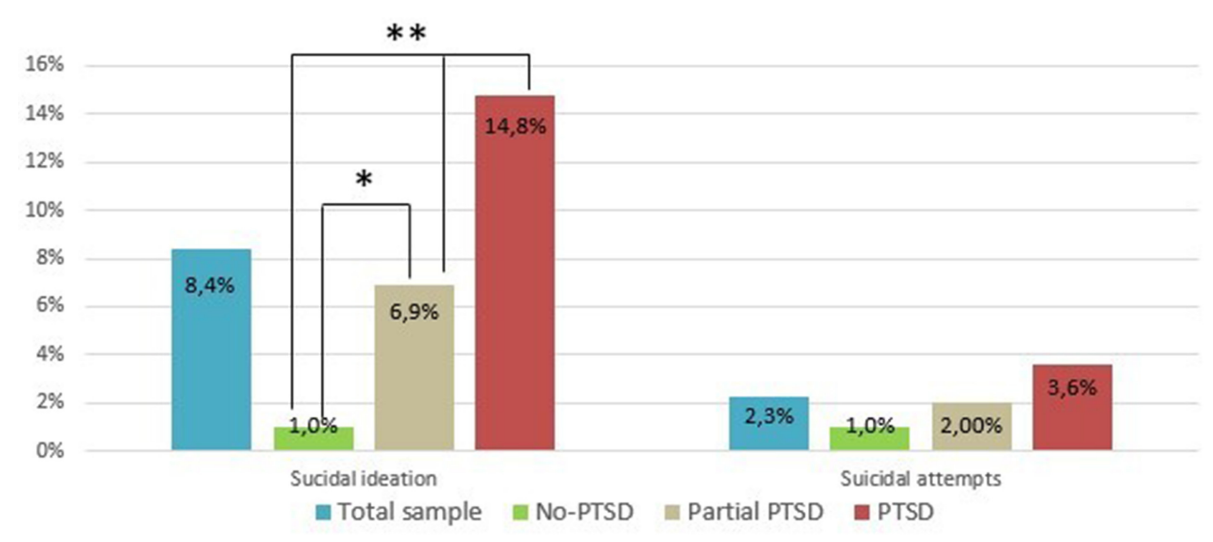

FIGURE 1 | Suicidal ideation and suicidal attempts rates in the total sample ( $N=475)$, No-PTSD $(N=104)$, partial PTSD $(N=202)$, and PTSD $(N=169)$ groups. *significantly higher rates respect to No-PTSD $(p=0.022)$. ${ }^{\star}$ significantly higher rates respect to partial PTSD $(p=0.014)$ and No-PTSD $(p<0.001)$.

TABLE 1 | TALS-SR domain scores in survivors with or without suicidal ideation amongst subjects with full or partial PTSD.

\begin{tabular}{|c|c|c|c|c|}
\hline TALS-SR domains & Total $(N=371)$ & No suicidal ideation $(N=332)$ & Suicidal ideation $(N=39)$ & $p$ \\
\hline (I) Loss events & $3.12 \pm 1.63$ & $3.09 \pm 1.62$ & $3.41 \pm 1.66$ & 0.247 \\
\hline (II) Grief-reactions & $10.13 \pm 5.32$ & $9.81 \pm 5.24$ & $12.82 \pm 5.32$ & 0.001 \\
\hline (III) Potential traumatic events & $3.44 \pm 2.45$ & $3.32 \pm 2.29$ & $4.43 \pm 3.42$ & 0.055 \\
\hline $\begin{array}{l}\text { (IV) Reactions to losses or traumatic } \\
\text { events }\end{array}$ & $8.12 \pm 2.99$ & $8.02 \pm 2.93$ & $8.97 \pm 3.36$ & 0.059 \\
\hline (V) Re-experiencing & $4.02 \pm 2.04$ & $3.94 \pm 1.99$ & $5.26 \pm 2.28$ & 0.037 \\
\hline (VI) Avoidance and numbing & $4.14 \pm 2.28$ & $4.01 \pm 2.25$ & $5.88 \pm 1.69$ & 0.001 \\
\hline (VII) Maladaptive coping & $1.04 \pm 1.39$ & $0.89 \pm 1.24$ & $2.28 \pm 1.86$ & $<0.001$ \\
\hline (VIII) Arousal & $2.60 \pm 1.44$ & $2.55 \pm 1.45$ & $2.97 \pm 1.27$ & 0.088 \\
\hline $\begin{array}{l}\text { (IX) Personal characteristics/risk } \\
\text { factors }\end{array}$ & $2.73 \pm 1.60$ & $2.66 \pm 1.58$ & $3.33 \pm 1.61$ & 0.013 \\
\hline
\end{tabular}

strongly support the notion that an exposure to a natural disaster, particularly to a high disruptive earthquake can eventually increase the risk for suicide behaviors and possibly promote a negative outcome in young subjects (11).

It is important to recall that we assessed survivors almost 2 years after the earthquake, so that this elapsed time might have also influenced the suicidality rates reported. In this regard, epidemiologic surveys conducted after 2 years from this event noted decreased rates of death by suicide with respect to the 5 years before the earthquake $(45,46)$. This is in line with previous research highlighting a reduction of the number of suicides in the 2 years post-disaster period. Conversely, we reported an increased number of suicides from the 3rd year after the catastrophe, during the "disillusionment" phase. This latter represents the period after 1 year or more after the disaster, when mental health problems can occur, also because of the disappointment and frustration toward the slow pace of resource allocation and reconstruction (45).

Taken together, our findings corroborate and extend the results of previous studies highlighting the role of PTSD as a relevant risk factor for suicidal ideation (47-50). In military veterans, PTSD diagnosis was associated to a six-fold increased risk of suicidal thought (51), and this relationship was also confirmed in civilian samples (49, 50, 52-54). Furthermore, PTSD individuals also resulted at higher risk for suicide attempts, especially if young or male $(16,54-57)$. On the contrary, no sexrelated differences were noted in our sample. As far as earthquake survivors are concerned, recent data pointed out an association between PTSD and suicidal attempts in 1,369 survivors 8 years after the disaster (41).

In addition to available findings, our study underlined a strong relationship between partial DSM-5 PTSD and suicide ideation. Previously, subthreshold PTSD symptoms were already associated with current suicidal ideation in a national survey on 9,358 subjects (58). This study was followed by another one on 252 earthquake survivors supporting the impact of partial PTSD on suicidality (3). A more recent study on 1,484 veterans described a relationship between DSM-5 partial PTSD and lifetime or current suicidal ideation (36). Our results strongly support the association between DSM-5 subthreshold PTSD symptoms and suicidal ideation, while confirming the relationship even in a civilian sample exposed to a mass trauma. On the contrary, suicidal attempts were not significantly related to full or partial PTSD in our sample, maybe for the small sample 
size and its relatively homogeneity and similar life habits of the subjects included that were all high-school senior students. In any case, to the best of our knowledge, there is a lack of studies in the literature about the role of subthreshold PTSD manifestation on the risk of suicide attempts, so that we are of the opinion that further studies in larger samples are warranted.

Four symptomatic TALS-SR domains, specifically, grief reactions, re-experiencing, avoidance and numbing, and maladaptive coping, were significantly higher in subjects with full-blow or partial PTSD reporting suicidal ideation, respect to those without this ideation. The relationship between PTSD symptom clusters and suicidal ideation is a primary topic for researchers and clinicians, in order to screen patients at higher risk for suicide and to develop targeted treatment strategies (59). Bereavement and pathological grief manifestations were related to PTSD severity and suicidality (60). Our data, exploring by the TALS-SR if the subjects had reported the loss of a close friend or relative during the earthquake that had caused the death of about 306 people in a little city, confirmed previous findings on the impact of a loss event on the post-traumatic stress reactions followed a mass trauma. Despite most of the previous studies on PTSD according to DSM-5 criteria showed an association between suicidal ideation and symptoms of hyperarousal, anhedonia, or negative affect (61-63), other researches reported contradictory results. A recent study also detected a relationship between suicidality and intrusive symptoms and avoidance, while presenting no hierarchy amongst PTSD symptom clusters on their specific impact on suicidal ideation (59). Furthermore, also externalizing manifestations, such as reckless or self-destructive behavior, alcohol or drug abuse and self-injuring are frequently observed in subjects with PTSD $(16,20,62,64)$. These symptoms, encompassed in the TALS-SR maladaptive coping domain, were related to suicidal ideation in our sample.

Several limitations of the study should be kept in mind when interpreting our results. As already mentioned, the first is the small sample size, although we attempted to recruit all existing high schools exposed to the earthquake. The second limitation is the use of self-report instruments, instead of clinical assessment that may be considered less accurate, and of no standardized and validated scale to assess suicide-related behaviors. The third is the lack of information about social support or Axis I psychiatric comorbidities that could affect suicidality, such as

\section{REFERENCES}

1. Farooqui M, Quadri SA, Suriya SS, Khan MA, Ovais M, Sohail Z, et al. Posttraumatic stress disorder: a serious post-earthquake complication. Trends Psychiatry Psychother. (2017) 39:135-43. doi: 10.1590/2237-6089-201 6-0029

2. Armenian HK, Morikawa M, Melkonian AK, Hovanesian A, Akiskal K, Akiskal HS. Risk factors for depression in the survivors of the 1988 earthquake in Armenia. J Urban Health. (2002) 79:373-82. doi: 10.1093/jurban/79.3.373

3. Lai TJ, Chang CM, Connor KM, Lee LC, Davidson JR. Full and partial PTSD among earthquake survivors in rural Taiwan. J Psychiatr Res. (2004) 38:313-22. doi: 10.1016/j.jpsychires. 2003.08.005 mood disorders $(22,52-54,65)$. Furthermore, data about preexistent psychiatric comorbidity and previous traumatic events are not available in our study. These variables, in fact, could influence peri-traumatic reactions during the earthquake or PTSD severity. Finally, the homogeneity of the study sample that included only non-clinical high school students and the cross sectional design of the study that did not allow to explore eventual changes in suicidality rates according to time after the exposure. However, it is also true that the homogeneity of the sample might also be a strength of the study.

In conclusion, our study indicates that both full and partial PTSD young subjects might be at risk for suicidal ideation, indicating the importance of evaluating and possibly treating even the subthreshold post-traumatic stress manifestations in survivors of a natural disaster. Since several symptoms, such as grief manifestations, re-experiencing, active avoidance, numbing, and maladaptive behaviors were related to suicidal thoughts, they should represent primary targets for interventions in the framework of PTSD management.

\section{DATA AVAILABILITY STATEMENT}

The data supporting the findings of the article are not publicly available, but it can be provided by the corresponding author on reasonable request.

\section{ETHICS STATEMENT}

The studies involving human participants were reviewed and approved by Ethics Committee of the University of L'Aquila. Written informed consent to participate in this study was provided by the participants' legal guardian/next of kin.

\section{AUTHOR CONTRIBUTIONS}

CC and LD participated to the conception and design of the study. $\mathrm{CC}, \mathrm{CB}$, and $\mathrm{LD}$ participated to the interpretation of the data, the draft, and critical revision of this article. $\mathrm{CB}$ undertook the statistical analysis. VD, CL, RR, and DM participated to the critical revision of the manuscript. All authors agreed to be cited as co-authors, accepting the order of authorship, and approved the final version of manuscript and the manuscript submission to Frontiers in Psychiatry.
4. Dell'Osso L, Carmassi C, Massimetti G, Daneluzzo E, Di Tommaso S, Rossi A. Full and partial PTSD among young adult survivors 10 months after the L'Aquila 2009 earthquake: gender differences. J Affect Disord. (2011) 131:79-83. doi: 10.1016/j.jad.2010.11.023

5. Zhang Z, Ran MS, Li YH, Ou GJ, Gong RR, Li RH, et al. Prevalence of posttraumatic stress disorder among adolescents after the Wenchuan earthquake in China. Psychol Med. (2012) 42:1687-93. doi: 10.1017/S0033291711002844

6. Carmassi C, Akiskal HS, Yong SS, Stratta P, Calderani E, Massimetti E, et al. Post-traumatic stress disorder in DSM-5: estimates of prevalence and criteria comparison versus DSM-IV-TR in a non-clinical sample of earthquake survivors. J Affect Disord. (2013) 151:843-8. doi: 10.1016/j.jad.2013.07.020

7. Gerstner RMF, Lara-Lara F, Vasconez E, Viscor G, Jarrin JD, OrtizPrado E. Earthquake-related stressors associated with suicidality, 
depression, anxiety and post-traumatic stress in adolescents from Muisne after the earthquake 2016 in Ecuador. BMC Psychiatry. (2020) 20:347. doi: 10.1186/s12888-020-02759-x

8. Gigantesco A, D’Argenio P, Cofini V, Mancini C, Minardi V. Health-related quality of life in the aftermath of the L'Aquila earthquake in Italy. Disaster Med Public Health Prep. (2016) 10:11-5. doi: 10.1017/dmp.2015.91

9. Fujiwara T, Yagi J, Homma H, Mashiko H, Nagao K, Okuyama M, et al. Suicide risk among young children after the Great East Japan Earthquake: a follow-up study. Psychiatry Res. (2017) 253:318-24. doi: 10.1016/j.psychres.2017.04.018

10. Jafari H, Heidari M, Heidari S, Sayfouri N. Risk factors for suicidal behaviours after natural disasters: a systematic review. Malays J Med Sci. (2020) 27:2033. doi: $10.21315 / \mathrm{mjms} 2020.27 .3 .3$

11. Kõlves K, Kõlves KE, De Leo D. Natural disasters and suicidal behaviours: a systematic literature review. J Affect Disord. (2013) 146:1-14. doi: 10.1016/j.jad.2012.07.037

12. Wagenaar BH, Hagaman AK, Kaiser BN, McLean KE, Kohrt BA. Depression, suicidal ideation, and associated factors: a cross-sectional study in rural Haiti. BMC Psychiatry. (2012) 12:149. doi: 10.1186/1471-244X-12-149

13. Matsubayashi T1, Sawada Y, Ueda M. Natural disasters and suicide: evidence from Japan. Soc Sci Med. (2013) 82:12633. doi: 10.1016/j.socscimed.2012.12.021

14. Stratta P, Capanna C, Riccardi I, Carmassi C, Piccinni A, Dell'Osso L, et al. Suicidal intention and negative spiritual coping one year after the earthquake of L'Aquila (Italy). J Affect Disord. (2012) 136:122731. doi: $10.1016 /$ j.jad.2011.10.006

15. Ozdemir O, Boysan M, Guzel Ozdemir P, Yilmaz E. Relationships between posttraumatic stress disorder (PTSD), dissociation, quality of life, hopelessness, and suicidal ideation among earthquake survivors. Psychiatry Res. (2015) 228:598-605. doi: 10.1016/j.psychres.2015.05.045

16. Carmassi C, Stratta P, Massimetti G, Bertelloni CA, Conversano C, Cremone IM, et al. New DSM-5 maladaptive symptoms in PTSD: gender differences and correlations with mood spectrum symptoms in a sample of high school students following survival of an earthquake. Ann Gen Psychiatry. (2014) 13:28. doi: 10.1186/s12991-014-0028-9

17. Arnberg FK, Gudmundsdóttir R, Butwicka A, Fang F, Lichtenstein P, Hultman $\mathrm{CM}$, et al. Psychiatric disorders and suicide attempts in Swedish survivors of the 2004 southeast Asia tsunami: a 5 year matched cohort study. Lancet Psychiatry. (2015) 2:817-24. doi: 10.1016/S2215-0366(15)00124-8

18. Ying L, Chen C, Lin C, Greenberger E, Wu X, Jiang L. The relationship between posttraumatic stress symptoms and suicide ideation among child survivors following the Wenchuan earthquake. Suicide Life Threat Behav. (2015) 45:230-42. doi: 10.1111/sltb.12118

19. Guo J, Liu C, Kong D, Solomon P, Fu M. The relationship between PTSD and suicidality among Wenchuan earthquake survivors: The role of PTG and social support. J Affect Disord. (2018) 235:90-5. doi: 10.1016/j.jad.2018.04.030

20. Dell'Osso L, Carmassi C, Stratta P, Massimetti G, Akiskal KK, Akiskal HS, et al. Gender differences in the relationship between maladaptive behaviors and post-traumatic stress disorder. A Study on 900 L' Aquila 2009 earthquake survivors. Front Psychiatry. (2013) 3:111. doi: 10.3389/fpsyt.2012.00111

21. Stratta P, Capanna C, Carmassi C, Patriarca S, Di Emidio G, Riccardi I, et al. The adolescent emotional coping after an earthquake: a risk factor for suicidal ideation. J Adolesc. (2014) 37:605-11. doi: 10.1016/j.adolescence.2014. 03.015

22. Carmassi C, Stratta P, Calderani E, Bertelloni CA, Menichini M, Massimetti E, et al. Impact of mood spectrum spirituality and mysticism symptoms on suicidality in earthquake survivors with PTSD. J Relig Health. (2016) 55:641-9. doi: 10.1007/s10943-015-0072-z

23. American Psychiatric Association. Diagnostic and Statistical Manual of Mental Disorders, 5th edn. Arlington, VA: American Psychiatric Publishing (2013).

24. Grubaugh AL, Magruder KM, Waldrop AE, Elhai JD, Knapp RG, Frueh BC. Subthreshold PTSD in primary care: prevalence, psychiatric disorders, healthcare use, and functional status. J Nerv Ment Dis. (2005) 193:658-64. doi: 10.1097/01.nmd.0000180740. 02644.ab

25. Dell'Osso L, Shear MK, Carmassi C, Rucci P, Maser JD, Frank E, et al. Validity and reliability of the structured clinical interview for the trauma and loss spectrum (SCI-TALS). Clin Pract Epidemol Ment Health. (2008) 4:2. doi: 10.1186/1745-0179-4-2
26. Breslau N, Lucia VC, Davis GC. Partial PTSD versus full PTSD: an empirical examination of associated impairment. Psychol Med. (2004) 34:120514. doi: 10.1017/S0033291704002594

27. McLaughlin KA, Koenen KC, Friedman MJ, Ruscio AM, Karam EG, Shahly $\mathrm{V}$, et al. Subthreshold posttraumatic stress disorder in the world health organization world mental health surveys. Biol Psychiatry. (2015) 77:37584. doi: 10.1016/j.biopsych.2014.03.028

28. Fink DS, Gradus JL, Keyes KM, Calabrese JR, Liberzon I, Tamburrino MB, et al. Subthreshold PTSD and PTSD in a prospective-longitudinal cohort of military personnel: potential targets for preventive interventions. Depress Anxiety. (2018) 35:1048-55. doi: 10.1002/da.22819

29. Naylor JC, Dolber TR, Strauss JL, Kilts JD, Strauman TJ, Bradford DW, et al. A pilot randomized controlled trial with paroxetine for subthreshold PTSD in Operation Enduring Freedom/Operation Iraqi Freedom era veterans. Psychiatry Res. (2013) 206:318-20. doi: 10.1016/j.psychres.2012.11.008

30. Meentken MG, van Beynum IM, Aendekerk EWC, Legerstee JS, El Marroun $\mathrm{H}$, van der Ende J, et al. Eye movement desensitization and reprocessing (EMDR) in children and adolescents with subthreshold PTSD after medically related trauma: design of a randomized controlled trial. Eur J Psychotraumatol. (2018) 9:1536287. doi: 10.1080/20008198.2018.1536287

31. Bergman HE, Kline AC, Feeny NC, Zoellner LA. Examining PTSD treatment choice among individuals with subthreshold PTSD. Behav Res Ther. (2015) 73:33-41. doi: 10.1016/j.brat.2015.07.010

32. Jakupcak M, Hoerster KD, Varra A, Vannoy S, Felker B, Hunt S. Hopelessness and suicidal ideation in Iraq and Afghanistan War Veterans reporting subthreshold and threshold posttraumatic stress disorder. J Nerv Ment Dis. (2011) 199:272-5. doi: 10.1097/NMD.0b013e3182124604

33. Dell'Osso L, Carmassi C, Rucci P, Conversano C, Shear MK, Calugi S, et al. A multidimensional spectrum approach to post-traumatic stress disorder: comparison between the Structured Clinical Interview for Trauma and Loss Spectrum (SCI-TALS) and the Self-Report instrument (TALS-SR). Compr Psychiatry. (2009) 50:485-90. doi: 10.1016/j.comppsych.2008.11.006

34. Dell'Osso L, Armani A, Rucci P, Frank E, Fagiolini A, Corretti G, et al. Measuring mood spectrum: comparison of interview (SCI-MOODS) and self-report (MOODS-SR) instruments. Compr Psychiatry. (2002) 43:6973. doi: 10.1053/comp.2002.29852

35. Carmassi C, Corsi M, Gesi C, Bertelloni CA, Faggioni F, Calderani E, et al. DSM-5 criteria for PTSD in parents of pediatric patients with epilepsy: what are the changes with respect to DSM-IV-TR? Epilepsy Behav. (2017) 70:97-103. doi: 10.1016/j.yebeh.2017.02.025

36. Mota NP, Tsai J, Sareen J, Marx BP, Wisco BE, Harpaz-Rotem I, et al. High burden of subthreshold DSM-5 post-traumatic stress disorder in U.S. military veterans. World Psychiatry. (2016) 15:185-6. doi: 10.1002/wps.20313

37. Chen CC, Yeh TL, Yang YK, Chen SJ, Lee IH, Fu LS, et al. Psychiatric morbidity and post-traumatic symptoms among survivors in the early stage following the 1999 earthquake in Taiwan. Psychiatry Res. (2001) 105:1322. doi: 10.1016/S0165-1781(01)00334-1

38. Vehid HE, Alyanak B, Eksi A. Suicide ideation after the 1999 earthquake in Marmara, Turkey. Tohoku J Exp Med. (2006) 208:19-24. doi: 10.1620/tjem.208.19

39. Lau JT, Yu X, Zhang J, Mak WW, Choi KC, Lui WW, et al. Psychological distress among adolescents in Chengdu, Sichuan at 1 month after the 2008 Sichuan earthquake. J Urban Health. (2010) 87:50423. doi: 10.1007/s11524-010-9447-3

40. Suzuki Y, Tsutsumi A, Fukasawa M, Honma H, Someya T, Kim Y. Prevalence of mental disorders and suicidal thoughts among community-dwelling elderly adults 3 years after the niigata-chuetsu earthquake. J Epidemiol. (2011) 21:144-50. doi: 10.2188/jea.JE20100093

41. Guo J, He H, Fu M, Han Z, Qu Z, Wang X, et al. Suicidality associated with PTSD, depression, and disaster recovery status among adult survivors 8 years after the 2008 Wenchuan earthquake in China. Psychiatry Res. (2017) 253:383-90. doi: 10.1016/j.psychres.2017.04.022

42. Kar N. Psychosocial issues following a natural disaster in a developing country: a qualitative longitudinal observational study. Int J Disas Med. (2006) 4:169-76. doi: 10.1080/15031430701875551

43. Kessler RC, Galea S, Jones RT, Parker HA, Hurricane Katrina Community Advisory Group. Mental illness and suicidality after Hurricane Katrina. Bull World Health Organ. (2006) 84:930-9. doi: 10.2471/BLT.06.033019 
44. Kessler RC, Galea S, Gruber MJ, Sampson NA, Ursano RJ, Wessely S. Trends in mental illness and suicidality after Hurricane Katrina. Mol Psychiatry. (2008) 13:374-84. doi: 10.1038/sj.mp.4002119

45. Stratta P, Rossi A. Suicide in the aftermath of the L'Aquila (Italy) earthquake. Crisis. (2013) 34:142-4. doi: 10.1027/0227-5910/a000166

46. Stratta P, Rossi A. Misreporting of suicide after the L'Aquila earthquake. BMJ. (2013) 346:f3179. doi: 10.1136/bmj.f3179

47. Panagioti M, Gooding P, Tarrier N. Post-traumatic stress disorder and suicidal behavior: a narrative review. Clin Psychol Rev. (2009) 29:47182. doi: 10.1016/j.cpr.2009.05.001

48. Krysinska K, Lester D. Post-traumatic stress disorder and suicide risk: a systematic review. Arch Suicide Res. (2010) 14:1-23. doi: 10.1080/13811110903478997

49. Bryan CJ. Treating PTSD within the context of heightened suicide risk. Curr Psychiatry Rep. (2016) 18:73. doi: 10.1007/s11920-016-0708-z

50. Boffa JW, Stanley IH, Hom MA, Norr AM, Joiner TE, Schmidt NB. PTSD symptoms and suicidal thoughts and behaviors among firefighters. J Psychiatr Res. (2017) 84:277-83. doi: 10.1016/j.jpsychires.2016. 10.014

51. Guerra VS, Calhoun PS, Mid-Atlantic Mental Illness Research, Education and Clinical Center Workgroup. Examining the relation between posttraumatic stress disorder and suicidal ideation in an OEF/OIF veteran sample. J Anxiety Disord. (2011) 25:12-8. doi: 10.1016/j.janxdis.2010.06.025

52. Carmassi C, Bertelloni CA, Dell'Oste V, Foghi C, Diadema E, Cordone A, et al. Post-traumatic stress burden in a sample of hospitalized patients with Bipolar Disorder: which impact on clinical correlates and suicidal risk? J Affect Disord. (2020) 262:267-72. doi: 10.1016/j.jad.2019.10.044

53. Dell'Osso L, Massimetti G, Conversano C, Bertelloni CA, Carta MG, Ricca V, et al. Alterations in circadian/seasonal rhythms and vegetative functions are related to suicidality in DSM-5 PTSD. BMC Psychiatry. (2014) 14:352. doi: 10.1186/s12888-014-0352-2

54. Panagioti M, Gooding PA, Triantafyllou K, Tarrier N. Suicidality and posttraumatic stress disorder (PTSD) in adolescents: a systematic review and meta-analysis. Soc Psychiatry Psychiatr Epidemiol. (2015) 50:52537. doi: 10.1007/s00127-014-0978-x

55. Brown LA, Armey MA, Sejourne C, Miller IW, Weinstock LM. Trauma history is associated with prior suicide attempt history in hospitalized patients with major depressive disorder. Psychiatry Res. (2016) 243:1917. doi: 10.1016/j.psychres.2016.06.046

56. LeBouthillier DM, McMillan KA, Thibodeau MA, Asmundson GJ. Types and number of traumas associated with suicidal ideation and suicide attempts in PTSD: findings from a U.S. Nationally Representative Sample. J Trauma Stress. (2015) 28:183-90. doi: 10.1002/jts.22010

57. Carrà G, Bartoli F, Carretta D, Crocamo C, Bozzetti A, Clerici M, et al. The prevalence of metabolic syndromein people with severe mental illness: a mediation analysis. Soc Psychiatry Psychiatr Epidemiol. (2014) 49:173946. doi: 10.1007/s00127-014-0835-y

58. Marshall RD, Olfson M, Hellman F, Blanco C, Guardino M, Struening EL. Comorbidity, impairment, and suicidality in subthreshold PTSD. Am J Psychiatry. (2001) 158:1467-73. doi: 10.1176/appi.ajp.158.9.1467

59. Brown LA, Contractor A, Benhamou K. Posttraumatic stress disorder clusters and suicidal ideation. Psychiatry Res. (2018) 270:238-45. doi: 10.1016/j.psychres.2018.09.030

60. Dell'Osso L, Carmassi C, Massimetti G, Conversano C, Daneluzzo E, Riccardi I, et al. Impact of traumatic loss on post-traumatic spectrum symptoms in high school students after the L'Aquila 2009 earthquake in Italy. J Affect Disord. (2011) 134:59-64. doi: 10.1016/j.jad.2011. 06.025

61. Guina J, Nahhas RW, Mata N, Farnsworth S. Which posttraumatic stress disorder symptoms, trauma types, and substances correlate with suicide attempts in trauma survivors? Prim Care Companion CNS Disord. (2017) 19:17m02177. doi: 10.4088/PCC.17m02177

62. Carmassi C, Bertelloni CA, Gesi C, Conversano C, Stratta P, Massimetti G, et al. New DSM-5 PTSD guilt and shame symptoms among Italian earthquake survivors: impact on maladaptive behaviors. Psychiatry Res. (2017) 251:1427. doi: 10.1016/j.psychres.2016.11.026

63. Spitzer EG, Zuromski KL, Davis MT, Witte TK, Weathers F. Posttraumatic stress disorder symptom clusters and acquired capability for suicide: a reexamination using DSM-5 criteria. Suicide Life Threat Behav. (2018) 48:10515. doi: $10.1111 /$ sltb. 12341

64. Carrà G, Scioli R, Monti MC, Marinoni A. Severity profiles of substanceabusing patients in Italian community addiction facilities: influence of psychiatric concurrent disorders. Eur Addict Res. (2006) 12:96101. doi: $10.1159 / 000090429$

65. Dell'osso L, Carmassi C, Rucci P, Ciapparelli A, Paggini R, Ramacciotti $\mathrm{CE}$, et al. Lifetime subthreshold mania is related to suicidality in posttraumatic stress disorder. CNS Spectr. (2009) 14:262-6. doi: $10.1017 /$ s1092852900025426.

Conflict of Interest: The authors declare that the research was conducted in the absence of any commercial or financial relationships that could be construed as a potential conflict of interest.

Copyright (c) 2021 Carmassi, Bertelloni, Dell'Oste, Luperini, Marazziti, Rossi and Dell'Osso. This is an open-access article distributed under the terms of the Creative Commons Attribution License (CC BY). The use, distribution or reproduction in other forums is permitted, provided the original author(s) and the copyright owner(s) are credited and that the original publication in this journal is cited, in accordance with accepted academic practice. No use, distribution or reproduction is permitted which does not comply with these terms. 